Fecha de recepción: septiembre 2019 Fecha de aceptación: noviembre 2019 Versión final: febrero 2020

\section{Del prototipo al producto: experiencia piloto para promover el emprendimiento temprano}

María Bernardita Brancoli ${ }^{(1)}$

Resumen: El proyecto "Del prototipo al producto" es una experiencia que se insertó en el pregrado de la carrera de Diseño de la Universidad del Desarrollo, como una actividad extracurricular para promover la comercialización temprana de diseños producidos por alumnos. El objetivo de esta iniciativa fue someter a los estudiantes en un proceso productivo real, uniendo una instancia académica con una práctica de emprendimiento. Esto implicó, entre otros aspectos, que a los alumnos se les enseñara que para comercializar un prototipo es necesario someterlo a un nuevo proceso de diseño que implica una evaluación de costo de producción, lo que trae consigo el sacrificio de decisiones de diseño que ellos no están acostumbrados a realizar. Esta experiencia dio como resultado la primera colección de objetos comercializables que reunió a estudiantes de diferentes talleres y sedes de la mención de Diseño de Ambientes y Objetos de la carrera de Diseño de la Facultad de Diseño, de la Universidad del Desarrollo.

Palabras clave: Diseño - desarrollo de productos - emprendimiento - colección.

[Resúmenes en inglés y portugués en la página 66]

(1) Diseñadora de la Pontificia Universidad Católica de Chile. Magister en Historia y Gestión de Patrimonio Universidad de los Andes. Diploma en Diseño Tipográfico, Pontificia Universidad Católica de Chile. Docente de la Facultad de Diseño Universidad del Desarrollo. Directora de Extensión, Comunicaciones y Centro de Diseño de la misma Facultad. Editora de la revista Base, Diseño e Innovación de diseño UDD.

Nuestra Facultad en su interés de dar visibilidad y proyección en circuitos nacionales e internacionales el trabajo de sus alumnos ha buscado permanentemente nuevos espacios para la difusión de sus proyectos. Esta ha organizado en los últimos años más de 16 exposiciones en recintos fuera del espacio académico mostrando el resultado de sus estudiantes. Dentro de estas exhibiciones ha participado con tres muestras en el Salón Satélite de Milán -instancia de promoción internacional de jóvenes talentos menores de 35 años y parte del Salón del Mueble de Milán-. En abril del año 2017, el Salón Satélite de Milán celebró 20 años de historia. En ese marco, se invitó solo a instituciones académicas 
que hubieran tenido una participación destacada en versiones anteriores, sometiéndolas a una rigurosa selección. La directora y curadora de este relevante evento, Marva Griffin, convocó a la Facultad de Diseño de la Universidad del Desarrollo debido a las muestras que se presentaron en las versiones 2011 y 2013 del Salón.

Esta invitación motivó a la Facultad, junto a la dirección de la mención Ambientes y Objetos, a crear un concurso interno de participación de alumnos, cuyo proceso duró dos semestres académicos. En el primer semestre participaron tres talleres de Santiago y uno de Concepción, que trabajaron con la temática de la celebración y bajo el requerimiento de diseñar con madera y metal, ambos materiales característicos del paisaje y la productividad de nuestro país. Los más de 50 alumnos -guiados por ocho docentes- entregaron alrededor de 50 propuestas, que fueron sometidas a una rigurosa evaluación académica y posteriormente a un proceso curatorial por parte de la Dirección de la Facultad para su selección. El resultado de este proceso dio como resultado 19 prototipos, realizados por alumnos de cuarto y quinto año, de la mención de Diseño de Ambientes y Objetos, de las sedes de la ciudad de Santiago y Concepción.

Una vez seleccionados estos proyectos, se creó un taller "Milán” para los alumnos de Santiago y un proceso de tutoría para Concepción. Los estudiantes -mentoreados por sus profesores- trabajaron durante un semestre para afinar los diseños y sobre todo para generar prototipos de excelencia, dignos de ser exhibidos en la cumbre del diseño mundial. De esta manera, nacieron los objetos que dieron nombre a la muestra-Celebrating $\mathrm{Na}$ ture-, reuniendo un proceso reflexivo y experimental, diseñando bajo la temática de la "celebración" a través de la comida. Los prototipos de utensilios y/o accesorios para comer fueron desarrollados en maderas locales en combinación con metales como cobre, bronce y acero inoxidable, reuniendo en un producto la importancia de la industria forestal y minera en el sector productivo chileno. Los alumnos crearon una colección de líneas simples y depuradas producidos bajo procesos semiartesanales en combinación con sistemas digitales. Como requerimiento formal, además de la temática, los objetos tenían la restricción de que debiesen caber en una maleta de mano, con el fin de asegurar su traslado a Milán. Es así, como los 19 alumnos junto a sus objetos tuvieron la oportunidad de viajar a Italia y participar de la Semana del Diseño de Milán. Durante la muestra, cada alumno participante junto a sus diseños pudieron intercambiar experiencias con estudiantes de otras escuelas de Diseño y jóvenes expositores, además de buscadores de tendencias, posibles compradores y también con los visitantes al Salón para difundir sus productos y contribuir a promover el diseño nacional y regional: la Facultad fue la única escuela de Diseño latinoamericana seleccionada para esta celebración. Durante los seis días que dura el evento cada estudiante se expone, cobra autonomía y es un ejercicio profesional real el enfrentarse al diálogo con distintos públicos, culturas e idiomas. 


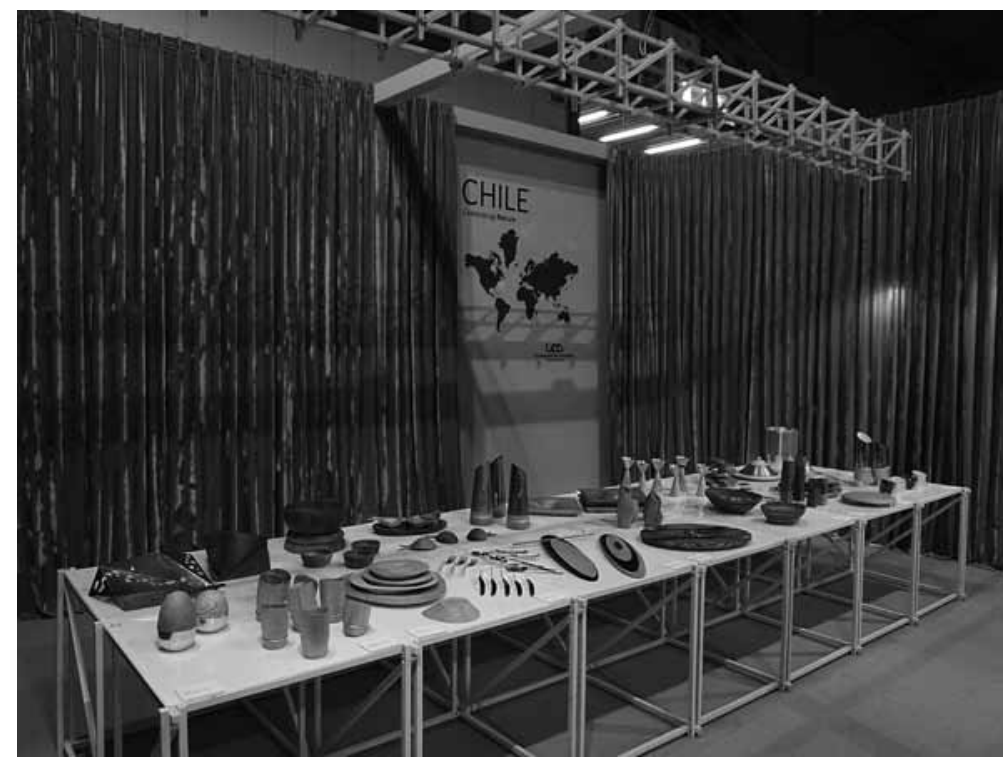

Figura 1. Stand de la muestra de diseño UDD presentada en el Salón Satélite de Milán.

En esta instancia los estudiantes son los protagonistas, generando compromiso con sus proyectos ya es una oportunidad de visibilización que les puede crear vínculos profesionales a largo plazo. Según el Plan de Fomento a la Economía Creativa, participar en estas instancias facilitan y fomentan la asociatividad entre los creadores, lo que genera un círculo virtuoso para la creación de nuevos proyectos. Sobre todo en países como el nuestro que geográficamente está alejado de los circuitos internacionales de diseño. Es así como nuestra Facultad se suma activamente a las estrategias gubernamentales de fomento para este sector productivo que para el año 2030, busca ser un motor de desarrollo social, cultural y económico del país realizando acciones de internacionalización del diseño nacional $\mathrm{y}$ fomentando la producción local.

\section{Del prototipo al producto}

A raíz de la participación en el Salón Satélite de Milán, nació la experiencia "Del prototipo al producto". La muestra generó un gran interés. Tanto que, una vez de regreso en Chile, motivados por las solicitudes internacionales y de algunas tiendas nacionales para 
adquirir los objetos, la Facultad decidió apoyar -sin fines de lucro-a los estudiantes en la creación de la primera colección de alumnos de Diseño UDD, disponible para la venta en el mercado nacional e internacional.

Para ello, se organizó una tutoría extracurricular con el objetivo de incentivar a los estudiantes a la comercialización de sus productos. Con este fin, se formó un equipo de tres docentes de ambas sedes: dos en Santiago y uno en Concepción que, por un período de tres meses, sostuvieron reuniones periódicas de trabajo con las 13 estudiantes que optaron por este camino, para revisar y evaluar cada uno de los diseños, sometiendo los prototipos a un proceso de edición y depuración para una futura producción en serie. El primer mes se sostuvieron reuniones semanales grupales e individuales y por vía streaming con los alumnos de Concepción para revisar cada uno de los diseños y buscar proveedores para la fabricación de los productos. El segundo mes se centró principalmente en la depuración de las formas para reducir costos y la sistematización de los tiempos de fabricación. El tercer mes se focalizó en el apoyo de fijación de precios para la comercialización de cada uno de los diseños. Los alumnos ayudados por sus tutores comprendieron empíricamente tanto la valorización de horas de diseño, costos de producción y marginación de utilidades como la elaboración de tablas de producción. Cada estudiante participante de esta colección tuvo que hacer iniciación de actividades económicas para poder cobrar por sus objetos.

\section{Educación para el emprendimiento}

Esta iniciativa se alinea con los propósitos de nuestra Facultad, que cuenta para estos fines con el Programa Red. Iniciado el año 2003, su propósito es mantener una vinculación con organizaciones públicas y privadas, que permita potenciar el aprendizaje experiencial en contextos reales a los alumnos, contribuyendo a la generación y transferencia de conocimiento desde la universidad a los distintos sectores productivos del país. Esto, además, se complementa con la malla curricular de la carrera de Diseño, que cuenta con una línea de Innovación y Emprendimiento con énfasis en el área de gestión y emprendimiento. Esta línea entrega a los estudiantes conceptos y habilidades orientadas a la búsqueda constante de oportunidades para crear e innovar, visualizando oportunidades para emprender desde el diseño. Si bien los conocimientos son entregados y evaluados durante el proceso de la carrera, es importante tener instancias de evaluación efectivas para poder aplicar estas competencias.

En el contexto educacional hay una creciente tendencia a incorporar como línea de formación el emprendimiento a sus estudiantes. Tener un vínculo con las empresas y organizaciones para enfrentarse a contextos reales es parte del proceso de aprendizaje en la mayoría de las carrera de diseño. Se ha observado que en el área de diseño y la mayoría de los egresados de Diseño UDD declaran que trabajan en sus propias empresas (Informe de la Marketing Inteligence Diseño UDD). Por otra parte en los informes del Ministerio de Educación generados por el Servicio de Información de Educación Superior (SIES), arroja que hay una tendencia a la baja en la empleabilidad de los egresados de la carrera de diseño en general. También se ha detectado mucha informalidad en el ejercicio de la 
profesión tanto como los empleadores como de los profesionales que prestan servicios, que no entregan boletas de honorarios, ni facturas ante el Servicio de Impuestos Internos por lo que no pueden ser contabilizados dentro de las estadísticas nacionales. Los estudios arrojan que existen 9.000 diseñadores ejerciendo de manera independiente y 2.200 empleados y alrededor de 38.000 profesionales del área (Mapeo de Industrias Creativas, 2012), donde el formato de ejercicio independiente es el más importante del sector. Es por esta razón que es necesario que nuestros egresados cuenten con mayores conocimientos y herramientas para dar valor a sus servicios y productos. Esto no sólo implica adquirir conocimientos en el área de la comercialización sino también comprender procesos sobre el registro de marcas, patentamiento y legislación de propiedad intelectual. Esto les facilitará en un futuro poder valorar el trabajo de ellos y el de otros y es un estímulo para la creación de empresas. Muchos emprendimientos se han transformado en una vía laboral importante para enfrentar, en muchos ámbitos, un ambiente laboral escaso y poco atractivo económicamente.

En el estudio de Mapeo de Industrias Creativas del año 2012 (Chile) se observó que en el sector creativo un $67 \%$ de los contribuyentes de este ámbito ejercen de manera independiente, esta cifra es superior a la de contribuyentes de otras disciplinas en el país. Igual tendencia se observa en la tributación empresas creativas, que en general la mayoría son micro empresas. Esto reafirma de un creciente interés en el ámbito del diseño de objetos por dedicarse o al menos intentar el desarrollo profesional por esta vía. El éxito de un proyecto depende en forma importante de la viabilidad del o los productos, pero también de una combinación de factores que no siempre están cubiertos dentro del aprendizaje de la carrera y se descubren-resuelven intuitivamente, no siempre con éxito.

Según Guerrero y Serey, en el Reporte Nacional de Chile 2018 (GEM, Chile), se necesitan condiciones para generar el emprendimiento como: apoyo financiero, políticas públicas, programas de gobierno, educación para el emprendimiento, transferencia de I+D, infraestructura profesional, apertura al mercado interno, acceso a la infraestructura física y normas civiles y culturales. Una de las mayores brechas de ellas en nuestro país es la educación para el emprendimiento que implica fomentar la creatividad, la iniciativa personal y el espíritu empresarial en todas las etapas del sistema educativo, siendo "las instituciones de formación superior y las universidades son las que proporcionan una formación adecuada y de calidad" (GEM NES, 2018). Nuestra universidad tiene incorporado en su modelo educativo las competencias de emprendimiento y liderazgo y autonomía, siendo estas transversales a todas las carreras dictadas en la universidad. Siguiendo esta línea en la carrera de diseño de Diseño UDD en su Perfil de Egreso y especialmente en el ciclo de habilitación profesional, estas competencias se ven reflejadas en los estudiantes ya que en este ciclo ellos están capacitados para "identificar oportunidades para innovar a través del diseño (...) fundamenta y desarrolla una propuesta con potencial para generar un emprendimiento (...) Cuantifica costos, identifica posibles fuentes de financiamiento y desarrolla un modelo de negocios que le permita implementar su propuesta, y en autonomía”. En esta misma línea, otras escuelas de diseño como la Facultad de Diseño y Comunicaciones de la Universidad de Palermo propone "diversas estrategias para ampliar el territorio del aula para generar nuevos entornos de aprendizaje e incorporando, desde una perspectiva innovadora, la mirada y estándares profesionales en el proceso formación 
de los estudiantes" (Echevarría, 2019). Otro ejemplo es la Escuela de Diseño de la Pontificia Universidad Católica de Chile que tiene dentro de su malla académica un curso de creación de nuevas empresas, donde los alumnos a lo largo del semestre deben presentar un plan de negocios para la creación de una nueva empresa. Todas estas iniciativas contribuyen desde la academia en la formación de futuros diseñadores emprendedores.

\section{Resultados}

El desarrollo de esta colección permitió a los estudiantes vivenciar un proceso cerrado de diseño, que incluyó iteración, contacto con proveedores, creación de planillas de venta para la fijación de precios competitivos en el mercado. Todo esto con el propósito de promover en ellos un espíritu de emprendimiento. También, les ayudó a comprender que para desarrollar y madurar un producto se requiere de mucho trabajo, tiempo y voluntad. En total, la duración de esta experiencia desde de ideación del diseño hasta que estuvo a la venta fue de aproximadamente 20 meses. Algunos alumnos ya estaban egresados en la etapa de comercialización de sus productos, pero el interés por ingresar en circuitos nacionales e internacionales los mantuvo vinculados a la universidad.

En paralelo, la Facultad contribuyó desarrollando una gráfica de apoyo para la venta de los productos. Para ello se realizaron fotografías de estudio para la difusión de los diseños y se elaboró un folleto y una línea de packaging para que los diseños pudieran ser comercializados.

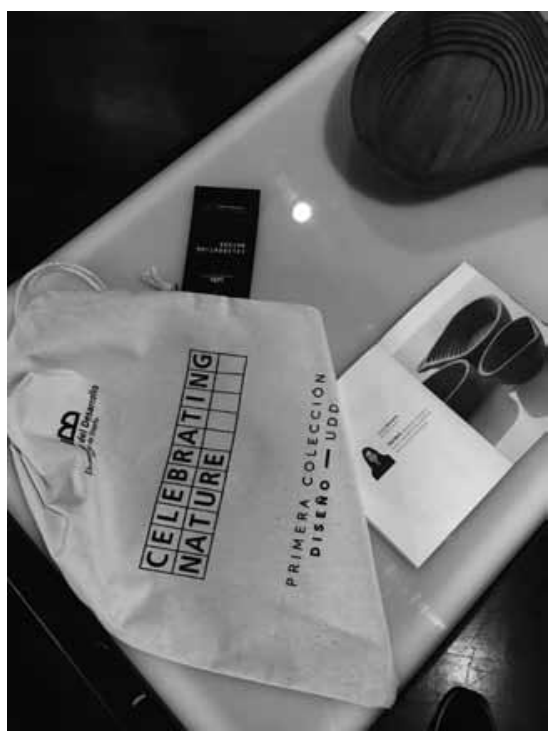

Figura 2. Imagen de la colección, folleto y etiquetas para la venta de productos. 
Los objetos fueron distribuidos en el Espacio Nacional de Diseño END, en el sitio web de Maison Numen en Miami y en el Aeropuerto privado Aviasur de Santiago. Las ganancias obtenidas de las ventas son íntegramente propiedad de los estudiantes. Como resultado de esta iniciativa algunas alumnas han emprendido y creado su propio negocio, creando su propia marca y nuevos diseños y están generando sus propia red de contactos para la comercialización de sus productos. Es el caso de Bewood de Javiera Vidal (www.bewood.cl). La experiencia de esta primera edición es un ejemplo de cómo se puede contribuir a fomentar el diseño nacional y crear alianzas para promover y comercializar desde el pregrado el trabajo de sus estudiantes fuera del aula. También esta iniciativa ayuda a los futuros profesionales a minimizar el riesgo transitando en el menor tiempo posible y con los menores baches en el camino desde la creación de un objeto hasta lograr instalar un producto con éxito en el mercado.

Este año nos encontramos realizando la segunda serie de objetos de Diseño UDD, esta vez en inspirados en la naturaleza extrema de nuestro país. Los alumnos de la mención de diseño de ambientes y objetos de ambas sedes se sometieron nuevamente a un riguroso proceso de diseño de dos semestres académicos con el fin de desarrollar una nueva colección diseños en cerámica para ser exhibidos y comercializados en la Semana del Diseño de Londres. Esta vez, el proceso de la cerámica permite seriar varias veces un objeto, facilitando su producción para su posterior comercialización. En esta versión, 21 alumnos tendrán la oportunidad de mostrar sus productos en un espacio gestionado por la Facultad de Diseño UDD dentro de la London Design Fair 2019. Paralelamente a la participación de los estudiantes en Londres, se está gestionando nuevamente el poder insertar esta colección dentro circuitos de venta a nivel nacional.

Creemos que estas instancias ayudan a aportar en la generación de nuevos diseñadores globalizados, inspirados, creativos y empresarios.

\section{Bibliografía}

Echavarría, O. (2019). “Preparación. Presentación: Proyección”. En XXXVII. 2019. Reflexión Académica en Diseño y Comunicación. Facultad de Diseño y Comunicación Universidad de Palermo, Vol 37, pág, 11.

Facultad de Diseño UDD. (2019) "Programa RED: Relación + Entorno + Diseño. Programa de vinculación con organizaciones públicas y privadas de la Facultad de Diseño de la Universidad del Desarrollo”.

Guerrero, M. y Serey, T. (2018) "Global Entrepeunership Monitor. Reporte Nacional de Chile 2018”. Universidad del Desarrollo.

Gobierno de Chile (2014). "Mapeo de Industrias Creativas, Caracterización y Dimensionamiento", Consejo Nacional de la Cultura y las Artes.

Gobierno de Chile (2017). "Plan de Nacional de Fomento a la Economía Creativa", Consejo Nacional de la Cultura y las Artes.

Ministerio de Educación, Gobierno de Chile https://www.mifuturo.cl/buscador-deempleabilidad-e-ingresos/. Consultado el 01 de julio de 2019. 
Universidad del Desarrollo (2018). "Informe de percepción de los alumnos de la Carrera de Diseño", Dirección de Marketing Intelligence.

Universidad del Desarrollo (2016). "Modelo Educativo Diseño UDD”.

\begin{abstract}
The project "From prototype to product" is an experience within the Design undergraduate studies at Universidad del Desarrollo, as an extracurricular activity to promote the early commercialization of designs produced by students. The objective of this initiative was to expose students to a real productive process, marrying an academic milestone with an entrepreneurial practice. This implied, among other aspects, that students should be taught that in order to market a prototype it is necessary to conduct a new design process that involves an evaluation of production costs, which brings with it the sacrifice of design decisions that they are not used to perform. This experience resulted in a first collection of marketable objects, which brought together students from different studios and venues of the Space and Object Design major, of the Design School of Universidad del Desarrollo.
\end{abstract}

Keywords: Design - product development - entrepreneurship - collection.

Resumo: O projeto "Do protótipo ao produto" é uma experiência que foi inserida na graduação da Universidade de Desenvolvimento da Universidade do Design, como uma atividade extracurricular para promover a comercialização precoce de projetos produzidos pelos alunos. O objetivo desta iniciativa era sujeitar os alunos a um processo produtivo real, unindo uma instância acadêmica a uma prática empreendedora. Isso implica, entre outros aspectos, que os alunos devem aprender que, para comercializar um protótipo, é necessário submetê-lo a um novo processo de design que envolve uma avaliação do custo de produção, o que traz consigo o sacrifício de decisões de design que eles não Eles são usados para executar. Essa experiência resultou na primeira coleção de objetos comercializáveis que reuniu estudantes de diferentes oficinas e locais com a menção de Design de Ambientes e Objetos da carreira de Design da Faculdade de Design da Universidade de Desenvolvimento.

Palavras chave: Desig - desenvolvimento de produtos - empreendedorismo - coleção.

[Las traducciones de los abstracts fueron supervisadas por el autor de cada artículo] 